\title{
Performance Comparison of Pan Tompkins and Wavelet Transform Based Ecg Feature Extraction Techniques
}

\author{
S N Shivappriya ${ }^{1}$, K. Maheswari ${ }^{2}$ and S. Sasikala ${ }^{3}$ \\ ${ }_{1,2,3}$ Kumaraguru College of technology, Coimbatore, India
}

\begin{abstract}
Electrocardiogram (ECG) signals represent the heart's electrical activity in terms of P-QRS-T wave components. It is necessary to denoise and extract the components from the raw ECG signal, which is fetched from the electrodes placed on the human chest. This work compares the Pan-Tompkins and Wavelet transform Technique for extracting predominant features from the ECG signals. The fiducial points like amplitude, time period, ECG signal's onset and offset points are detected based on the windowing, thresholding Techniques. The accurate delineation takes place with appropriate scaling and wavelet functions. The various performance parameters like sensitivity, positive predictivity and accuracy are used to compare these feature extraction methods. The accurate feature extraction will give the accurate information about the heart functioning, which will improve the exact detection of the ECG signal wave components, diagnosis, and treatment.
\end{abstract}

KEY WORDS: ECG, PAN TOMPKINS, WAVELET TRANSFORM, DETECTION, DELINEATION.

\section{INTRODUCTION}

Electro Cardiogram: Electro Cardio Gram (ECG) represents the human's heart's electrical activity, recorded by skin electrode. ECG signal pattern reflects the condition of the human heart [Bonow Libby \& Mann Zipes, 2006]. It is a noninvasive technique [0'Rourke, RA (Ed.) 2005], the electrodes are positioned on the human body through these electrodes the ECG signals are measured [Mathers CD et al., 2004]. If there is any variation in the morphological pattern of the ECG signal, indicates the abnormality in heart rate or rhythm, which is the reflection of the cardiac arrhythmia, these arrhythmias can be detected by analyzing from the taped ECG waveform. The physiological nature of the heart information is present

\section{ARTICLE INFORMATION}

*Corresponding Author: shivappriya.sn.ece@kct.ac.in Received 20th Oct 2020 Accepted after revision 11th Dec 2020 Print ISSN: 0974-6455 Online ISSN: 2321-4007 CODEN: BBRCBA

Thomson Reuters ISI Web of Science Clarivate Analytics USA and Crossref Indexed Journal

\section{Clarivate
Analytics}

NAAS Journal Score 2020 (4.31) SJIF: 2020 (7.728)

A Society of Science and Nature Publication,

Bhopal India 2020. All rights reserved.

Online Contents Available at: http//www.bbrc.in/

Doi: http://dx.doi.org/10.21786/bbrc/13.11/28 on the duration and amplitude of the ECG signal. The ECG wave represents the depolarization and repolarization of the ions in the myocardial tissue [Thom Thomas Thom 2006], which forms the P-QRS-T waves.

\section{MATERIAL AND METHODS}

Databases: PhysioBank [Physionet database 2010] is a library of biomedical signals, which can be used by the research society. PhysioBank has a collection of healthy persons ECG record and holds the patients with abnormal and acute condition records like Myocardial Infraction, Bundle Branch Blocks, Ventricular Tachycardia, Ventricular and Atrail Arrhythmias etc.,

Denoising Ecg Signal: Raw ECG signal fetched from the patient body consists of the following noises:

- Electrode movement noise

- Baseline wandering and drift

- Power line interference

- Muscle noise

- Channel noise

- Instrumentation noise 
With Finite Impulse Response (FIR) filter, Linear filter, Infinite Impulse Response (IIR) filter, Non-linear filter and the adaptive filtering techniques are used for removing the noises present in the raw ECG signal [Raimon Jané et al., 1992]-[ Seema Nayak et al., 2012]. These filters will have the drawback of time and frequency localization, which can be overcome with the Wavelet Transform based Denoising technique [Mikhled Alfaouri and Khaled Daqrouq 2008].

Feature Extraction: After Denoising the raw ECG signal from the noisy or distorted ECG signal. The extraction of the ECG signal components (P-QRS-T waves) is essential for the analysis. With the traditional rule based feature extraction technique, the features like amplitude, frequency onset and offset of the ECG wave elements are derived. [Issac Nivas et al., 2005] Shows how the efficient feature extraction technique will improve the performance of the automated detection and classification processes. This work shows that how the wavelet transform based delineation approach improves the performance of the Denoising process than the traditional Pantompkin's Algorithm Feature Extraction / Detection technique.

Pantompkin's Algorithm for Feature Extraction: Pan \& Tompkins et al., (1985) is the traditional feature extraction method used for getting features from the P-QRS-T waves. After filtering process, the derivation, squaring, and integration functions used to find out peaks like Q, P and T amplitudes. In addition to these two adaptive thresholding techniques used to predict the QRS complex. In some arrythmia's there will not be any QRS complex, in those situations search back algorithm has been used to detect the $\mathrm{P}$ and $\mathrm{T}$ wave. The flow diagram of Pan Tompkins Algorithm is shown below.

Figure 1: Pan Tompkins Algorithm for ECG Segmentation Flow diagram

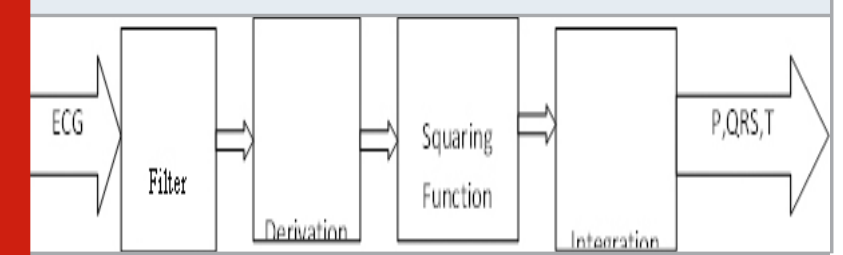

Band Pass (BP) filter: The concatenation of the Low and high Pass forms the Band Pass(BP) filter. The BP filter cutoff frequency is $5-15 \mathrm{~Hz}$, which is the energy frequency range of QRS complex. This band pass filter not only filters the ECG components and suppresses the noises like muscle artifacts, power-line disturbance, base-line drifting etc.,

Equation (1) shows the Low pass filter Transfer Function (Trans Flp)

Trans $\mathrm{F}_{\mathrm{lp}}(\mathrm{z})=\left(\mathrm{l}-\mathrm{z} 1^{-6}\right) 2 /\left(\mathrm{l}-\mathrm{z} 1^{-1}\right)^{2}$

$\mathrm{F}_{\mathrm{c}}=11 \mathrm{~Hz}$, Gain = 36. Equation (2) shows the difference operation $y d(n T)=2 y d(n T-T)-y d(n T-2 T)+x d(n T)-2 x d(n T-$

$6 \mathrm{~T})+\mathrm{xd}(\mathrm{nT}-12 \mathrm{~T})$

$\operatorname{TFlp}(\mathrm{z})=\left(1-\mathrm{z} 1-^{32}\right) /\left(1-\mathrm{z}^{-1}\right)$

$\operatorname{TFhp}(\mathrm{z})=\mathrm{z} 16-\operatorname{TFlp}(\mathrm{z} 1) / 32$

$\operatorname{TFhp}(\mathrm{z})=\left(-\mathrm{z} 1^{32}+32 \mathrm{z} 1^{16}-32 \mathrm{z} 1^{15}+\mathrm{l}\right) /$

$\left(32 z 1^{32}-32 z 1^{31}\right)$

$p(n T)=x d(n T-16 T)-0.0313[y d(n T-T)$

$+x d(n T)-x d(n T-32 T)]$

Lower Cut off Frequency $\left(\mathrm{F}_{\mathrm{c}}\right)=5 \mathrm{~Hz}$,

Gain $=1$ and delay is $80 \mathrm{~ms}$.

Derivative: ECG signal derivation shows the QRS complex slope. With the following transfer function.

Trans $\mathrm{F}(\mathrm{z})=0.1^{*}\left(2+\mathrm{z} 11-\mathrm{z} 1^{3}-2 \mathrm{z} 1^{4}\right)$

The Trans F's difference equation is:

$\mathrm{yd}(\mathrm{nT})=(1 / 8) *[2 \mathrm{xd}(\mathrm{nT})+\mathrm{xd}(\mathrm{nT}-\mathrm{T})-$

$x d(n T-3 T)-2 x d(n T-4 T)$

Squaring: The squaring of the signal shows higher positive values.

Figure 2: Block diagram of the ECG beat segmentation

\section{Signal Preprocessing}

Afarmlnt Nanmmnntitinn and

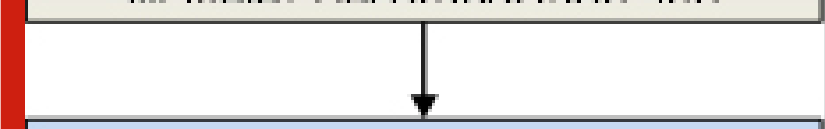

\section{Baseline shift elimination}

Mavolot Nolinoatinn ucing

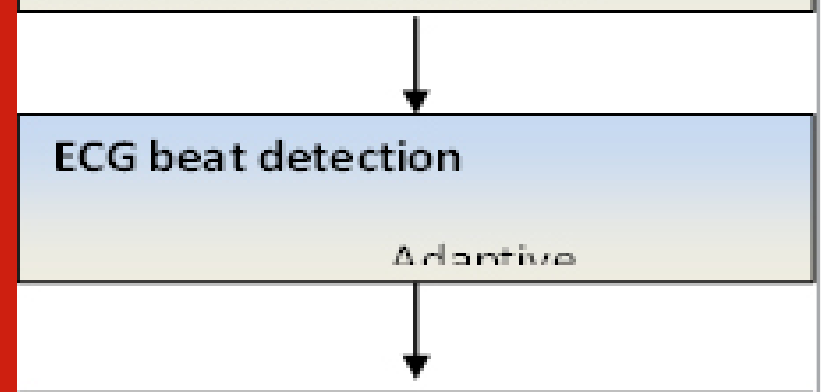

\section{ECG wave components}

Muiti_ Sralo M/avolot Foatiuroc

$\mathrm{yd}(\mathrm{nT})=[\mathrm{xd}(\mathrm{nT})] 2$

(9)

Moving integrator: The detection of $\mathrm{R}$ wave is not a supreme way to detect the QRS complex, in the abnormal ECG signal there may be much large amplitude and long 
duration QRS complexes. In those situations, the moving integrator used to detect those wave components. The difference equation of the moving integrator is:

$\mathrm{yd}(\mathrm{nT})=(1 / \mathrm{N})^{*}[(\mathrm{xd}(\mathrm{nT})-(\mathrm{N}-\mathrm{l}) \mathrm{T})+$

$(\mathrm{xd}(\mathrm{nT})-(\mathrm{N}-2) \mathrm{T})+\ldots+\mathrm{xd}(\mathrm{nT})]$

Width of the moving window integrator with $\mathrm{N}$ samples.

2.3.2 Wavelet Transform (WT) for Feature Extraction: Features play a vital role, for the evaluation of the ECG signal from the normal and abnormal patients. So, to get time, frequency and amplitude localized signal, the wavelet transform is used. Sankara Subramanian Arumugam et al., 2009 shows how the wavelet transform technique, is used for the detection of Cardiac arrhythmia signal. Figure 2 shows the ECG segmentation method the QRS complex from the P and T waves. For the MIT-BIH database the QRS complex detection rate is $99.8 \%$. It is essential to find the timing difference between the several QRS intervals than the subjective assessment of ECG morphology [Sahambi JS et al., 1997].

ECG Delineation for Feature Extraction: S.N. Shivappriya et al., (2006) shows how the detection and delineation process takes place with the Stationary Wavelet Transform (SWT). Martinez JP (2004) shows the evaluation of various datasets like MIT-BIH Arrhythmia, European ST-T and QT database. Natalia M Arzeno et al., (2009) Obtained only ( $\mathrm{Se}=99.68 \%, \mathrm{Pp}=99.63 \%)$ and the largest time error. The proposed wavelet-based delineation approach, for the QRS complex detection of MIT-BIH Arrhythmia Database shows the sensitivity ( $\mathrm{Se}=99.30 \%)$ and Positive Predictivity ( $\mathrm{PP}=99.39 \%)$. Laguna P et al., (1985) shows the Low Pass Differentiator (LPD) approach, which will not give.

faultless T-wave end point detection, as it has Low Pass Filter and differentiator, which is simple in implementation and robust to waveform variations. The WT overcomes the drawbacks of Low Pass Differentiator (LPD), in terms of sensitivity to noise and arbitrary threshold, and provides time-frequency- amplitude localization, represents temporal features with different resolution, which is the suitable technique for analyzing the ECG signal. Wavelet Based ECG Delineator used to detect the diverse morphologies of QRS complex, $\mathrm{P}$ and $\mathrm{T}$ waves with the generalization of the detection technique. [Laguna P et al., 1985] LPD method is compared with the proposed method: the T wave and $U$ wave detection and delineation performance is higher, particularly in the T wave end, and also locates different waves with different amplitudes in a more accurate manner.

Morphological Features: ECG morphology shows the series of deflections of heart muscle, which is away from the reference point on the ECG.

Morphological features:

1. QRS interval,

2. T wave interval,
3. P wave interval,

4. R amplitude,

5. $\mathrm{P}$ and $\mathrm{T}$ wave amplitude,

6. $\mathrm{R}$ and $\mathrm{S}$ amplitude,

7. QRS delineation interval,

8. T wave delineation interval,

9. $\mathrm{P}$ wave delineation interval

10. RR interval.

The proposed method uses the following medical metrics,

True Positive Rate (TPR) or Sensitivity

$$
(\mathrm{Se})=\frac{\mathrm{TP}}{\mathrm{TP}+\mathrm{FN}} \times 100
$$

Positive Predictive Value (PPV) or
Precision $=\frac{\mathrm{TP}}{\mathrm{TP}+\mathrm{FP}} \times 100$

Accuracy $=\frac{\mathrm{TP}+\mathrm{TN}}{\mathrm{TP}+\mathrm{FN}+\mathrm{FP}+\mathrm{TN}} \mathrm{X} 100$

True Positive (TP)- truly detected measures False Negative (FN)- erroneously rejected measures False Positive (FP)- mistakenly detected measures True Negative (TN)properly rejected measures.

\section{RESULTS AND DISCUSSIONS}

WT algorithm is utilized over the digitized and denoised ECG signal. The 21 to 25 scales are preferred which hold the predominant energy information. The QRS complex energy gets depleted when the scale is more than 24. Due to that 23 preferred for the detection of QRS complex, $\mathrm{P}$ and $\mathrm{T}$ wave is detected at 24 and 25 scale. Figure 3 shows the Wavelet Transformed and Noise removed ECG signal.

Figure 3: Shows that the Multi level (1 to 4 scale) wavelet transformed denosied ECG signal.

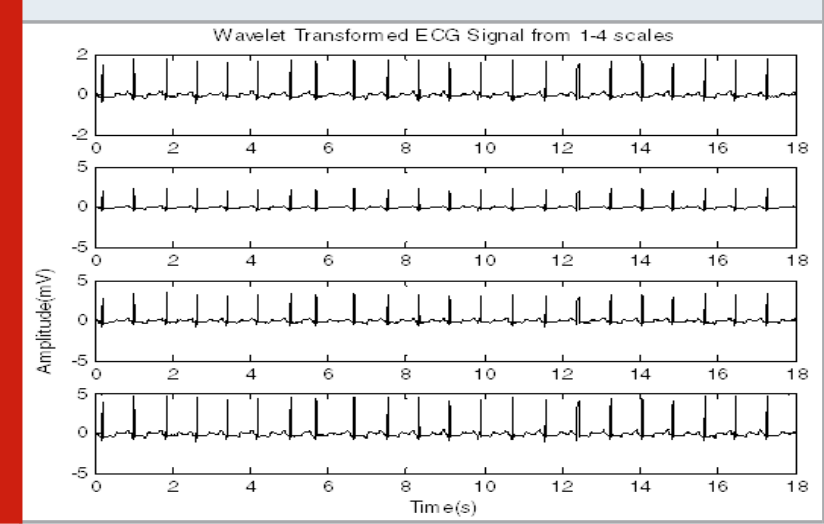

Performance Measures of Feature Extraction Process: From the MIT Data Base, QT Data Base and European Data Base 151 annotations are used for the investigation of the ECG signal. Figure 4 shows the Wavelet Transform based delineated RR intervals. Figure 5 \& 6 shows the 
performance measure of Pan Tompkins's detection method and wavelet-based delineation method.

The Sensitivity of the proposed approach is 99.83\% which is comparatively higher than the Low Pass Differentiator (LPD) method with $97.74 \%$ and Discrete Wavelet Transform (DWT) method with 99.77\% sensitivity. Costas Papaloukas et al., 2002 achieved only 80.09\% for the detection of T Wave episode and Se of 92.02\% and PP of 93.77\%. In the feature extraction process, different morphological and statistical parameters of P, QRS Complex and T wave of the ECG signals are extracted. Figure 6 shows the RR interval delineation time feature for all the individual ECG cycles of 1800 samples.

Figure 4: RR interval Delineation time Feature for 300 samples of 6 classes

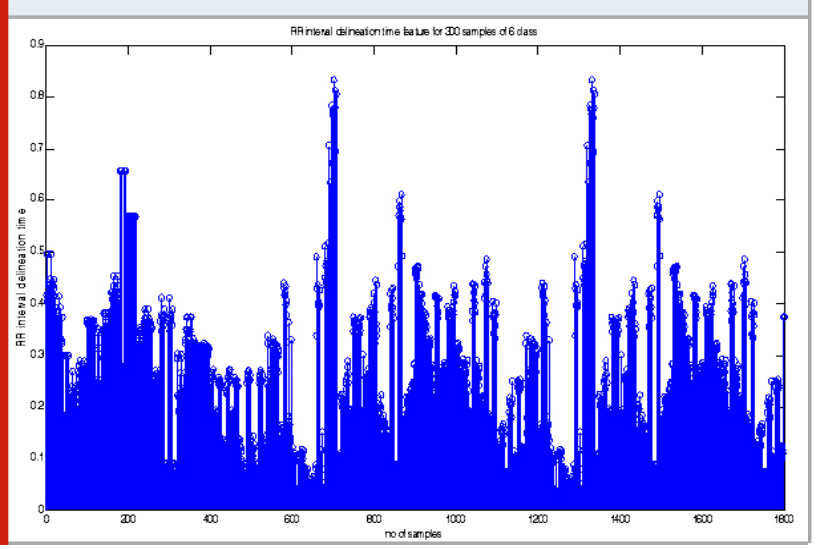

Figures 5: Pan Tompkin's detection method

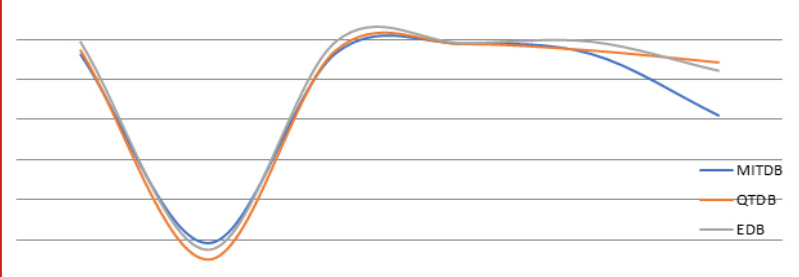

Figures 6: Wavelet Transform based delineation method

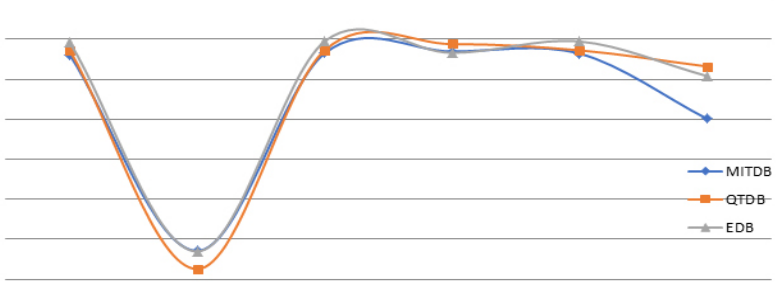

\section{CONCLUSION}

In ECG Monitoring System, for the analysis of several heart diseases, the detection of various features of the ECG signal is very important. This work compares the Pan Tompkins's and Wavelet Transform based feature extraction techniques for detection and delineation of ECG signal components like P-QRS-T waves. From the Feature Extraction Process, different morphological features of ECG signal are extracted : QRS detection \& delineation time, $\mathrm{R}$ wave detection $\&$ delineation time, $\mathrm{P}$ wave amplitude, $\mathrm{P}$ wave detection $\mathrm{Ct}$ delineation time, $\mathrm{R}$ amplitude, $\mathrm{S}$ amplitude, $\mathrm{T}$ amplitude, $\mathrm{T}$ wave detection Et delineation time, RR interval, Slope of ST interval. This work compares the performance of the finding and delineation process of the QRS complex, $\mathrm{T}$ and $\mathrm{P}$ waves with these measures' sensitivity and positive predictivity.

\section{ACKNOWLEDGEMENTS}

The authors thank the management and Principal of Kumaraguru College of Technology, Coimbatore for providing excellent computing facilities and encouragement. The author would also like to thank the Laboratory for Computational Physiology at MIT for providing the annotated data for this study.

\section{REFERENCES}

Bonow Libby \& Mann Zipes, 2006, Heart Disease: a textbook of cardiovascular medicine, eighth edition, Saunders, Elsevier.

Costas Papaloukas, Dimitrios I Fotiadis, Aristidis Likas, Christos S Stroumbis \&t Lampros K. Michalis, 2002, 'Use of a novel rule-based expert system in the detection of changes in the ST segment and the T wave in long duration ECGs', Journal of Electrocardiography, vol. 35, no. 1, pp. 27-34.

Issac Nivas \&t Shantha Selva kumari R 2005, 'Artificial neural network based automatic cardiac abnormalities classification', Computational Intelligence and Multimedia Applications, IEEE, pp. 41-46.

Laguna P, Thakor NV, Caminal P, Jane R, Yoon H-R, Luna AB, Pan J \& Tompkins WJ 1985, 'A real-time QRS detection algorithm', IEEE Transaction Biomedical Engineering, vol. 3, pp. 230-236.

Laguna P, Thakor NV, Caminal P, Jane R, Yoon H-R, Luna AB, Pan J \& Tompkins WJ 1985, 'A real-time QRS detection algorithm', IEEE Transaction Biomedical Engineering, vol. 3, pp. 230-236

Mahmoodabadi SZ, Ahmadian A \&t Abolhasani MD 2005, 'ECG feature extraction using Daubechies wavelets', In: Proc. Fifth IASTED International Conference, pp. 343348.

Martinez JP, Almeida R, Olmos S, Rocha AP \& Laguna P 2004, 'A Wavelet-Based ECG Delineator: Evaluation on Standard Databases', IEEE Transactions on Biomedical Engineering, vol. 51, no. 4, pp.571-581.

Mathers CD, Lopez A \& Stein D 2004, 'Deaths and disease 
burden by cause: Global burden of disease estimates by World Bank Country Groups' Lopez AD, Mathers CD, Ezzati M, et al., editors. Washington (DC).

Mikhled Alfaouri and Khaled Daqrouq 2008. 'ECG Signal Denoising By Wavelet Transform Thresholding', American Journal of Applied Sciences 5 (3): 276-281, 2008ISSN 1546-9239

Natalia M Arzeno, Zhi-De Deng \& Chi-Sang Poon 2009, 'Analysis of First-Derivative Based QRS Detection Algorithms', IEEE Trans Biomed Eng. Author manuscript; available in PMC, pp.478-484.

O'Rourke, RA (Ed.) 2005. 'Hurst's the Heart: Manual of Cardiology' (11th ed.) New York: McGraw-Hill, Medical Pub. Division

Physionet database 2010, online Available from <www. physionet.org/ physiobank/database $>$.

Raimon Jané, Pablo Laguna, Nitish V. Thakor \& Pere Caminal 1992, 'Adaptive Baseline Wander Removal in the ECG: Comparative Analysis with Cubic Spline Technique', Computers in Cardiology, vol.2, pp.143146.
S.N. Shivappriya, R. ShanthaSelvaKumari, T.GowriShankar. "ECG Delineation using Stationary Wavelet Transform”, 2006 International Conference on Advanced

Sahambi JS, Tandon S \& Bhatt RKP 1997, 'Using wavelet transforms for ECG characterization, an on-line digital signal processing system', IEEE Engineering in Medicine and Biology Magazine, vol.16, pp. 77-83.

Sankara Subramanian Arumugam, Gurusamy Gurusamy At Selvakumar Gopalasamy 2009, 'Wavelet based detection of ventricular arrhythmias with neural network classifier', JBISE, vol.2, no.6, pp.439-444.

Seema Nayak, Soni MK \& Dipali Bansal 2012, 'Filtering Techniques For ECG Signal Processing', International Journal of Research in Engineering and Applied Sciences, vol.2, no. 2, pp.671-679.

Thom Thomas Thom, Nancy Haase \& Wayne Rosamond, 2006, Heart disease and stroke statistics-2006 update. A report from the American heart association statistics committee and stroke statistics subcommittee, in circulation. 